\title{
Can bayesian models play a role in dental caries epidemiology? Evidence from an application to the BELCAP data set
}

\section{Domenica Matranga ${ }^{1}$, Alberto Firenze ${ }^{1}$ and Angela Vullo ${ }^{2}$}

${ }^{1}$ Sciences for Health Promotion and Mother and Child Care, University of Palermo,

Palermo, Italy, ${ }^{2}$ Economics, Business and Finance, University of Palermo, Palermo, Italy
Matranga D, Firenze A, Vullo A. Can bayesian models play a role in dental caries epidemiology? Evidence from an application to the BELCAP data set. Community Dent Oral Epidemiol 2013; 41: 473-480. (C) 2013 John Wiley \& Sons A/S. Published by John Wiley \& Sons Ltd

Abstract - Objectives: The aim of this study was to show the potential of Bayesian analysis in statistical modelling of dental caries data. Because of the bounded nature of the $\mathrm{dmft}$ (DMFT) index, zero-inflated binomial (ZIB) and beta-binomial (ZIBB) models were considered. The effects of incorporating prior information available about the parameters of models were also shown. Methods: The data set used in this study was the Belo Horizonte Caries Prevention (BELCAP) study (Böhning et al. (1999)), consisting of five variables collected among 797 Brazilian school children designed to evaluate four programmes for reducing caries. Only the eight primary molar teeth were considered in the data set. A data augmentation algorithm was used for estimation. Firstly, noninformative priors were used to express our lack of knowledge about the regression parameters. Secondly, prior information about the probability of being a structural zero $\mathrm{dmft}$ and the probability of being caries affected in the subpopulation of susceptible children was incorporated. Results: With noninformative priors, the best fitting model was the ZIBB. Education (OR $=0.76,95 \%$ CrI: $0.59,0.99)$, all interventions $(\mathrm{OR}=0.46,95 \%$ CrI: $0.35,0.62)$, rinsing (OR $=0.61,95 \% \mathrm{CrI}: 0.47,0.80)$ and hygiene $(\mathrm{OR}=0.65$, $95 \%$ CrI: $0.49,0.86)$ were demonstrated to be factors protecting children from being caries affected. Being male increased the probability of being caries diseased $(\mathrm{OR}=1.19,95 \% \mathrm{CrI}$ : 1.01, 1.42). However, after incorporating informative priors, ZIB models' estimates were not influenced, while ZIBB models reduced deviance and confirmed the association with all interventions and rinsing only. Discussion: In our application, Bayesian estimates showed a similar accuracy and precision than likelihood-based estimates, although they offered many computational advantages and the possibility of expressing all forms of uncertainty in terms of probability. The overdispersion parameter could expound why the introduction of prior information had significant effects on the parameters of the ZIBB model, while ZIB estimates remained unchanged. Finally, the best performance of ZIBB compared to the ZIB model was shown to catch overdispersion in data.
Key words: Bayesian analysis; Belo Horizonte Caries Prevention; bounded data; $\mathrm{dmft}$; informative prior; zero-inflated betabinomial; zero-inflated binomial

Domenica Matranga, Sciences for Health Promotion and Mother and Child Care, University of Palermo, Via del Vespro 133, Palermo, Sicily 90127, Italy

Tel.: +39 0916553604

Fax: +39 0916553647

e-mail: domenica.matranga@unipa.it

Submitted 14 November 2012; accepted 27 January 2013
Statistical models play a key role in epidemiology to provide the best representation of oral health data and interpretation of the data generation process. Final indicators deriving from each model's results are helpful for epidemiologist working in the oral health field, with the aim of achieving the goals set by international institutions such as the World Health Organization.

Bayesian and Frequentist are the two souls of statistical modelling. Frequentist probability is 
defined as the relative frequency of an event in hypothetical infinite sequences of randomized experiments or random samplings. The constant parameter defining the population under study is estimated under the theoretical distribution hypothesized for random error. As an alternative, Bayesian probability is defined as the 'personal belief' that a rationale and coherent subject may have upon the occurrence of an event. Within this setting, parameter is a random variable whose distribution, the so-called prior distribution, is used to express personal uncertainty about parameter's value. After data observation, Bayes' rule is applied to obtain posterior distributions for unknown parameters, which take into account both the priors and the data. In practice, the difference between the two approaches is the explicit prior used in Bayesian reasoning. The experimenters' knowledge or experience can be essential information, which makes the Bayesian inference more plausible. Sensitivity analysis is usually conducted to evaluate the robustness of results relative to the choice of the prior. As an alternative to informative priors, either empirical priors or vague priors can be chosen (1).

Bayesian analysis spread only after the algorithmic developments of the late 1980s. Using Markov chain Monte Carlo (MCMC) algorithms for Bayesian computation, it is straightforward to fit realistic and complex models, whose assessment of theoretical assumptions of maximum-likelihood (ML) estimation can be very difficult or impossible. One advantage of using MCMC procedures can be traced in the credibility interval (CrI)'s interpretation as the interval containing the true parameter with some probability (1- $\alpha)$. By contrast, interpretation of the Frequentist confidence interval is far less attractive as the interval containing the true parameter 100(1- $\alpha$ ) per cent of the time if data were repeatedly produced by the assumed random sampling process. Epidemiologic surveys are difficult to frame within a repeated sampling scheme, which makes the Bayesian interpretation more appealing. Frequentist hypotheses are questionable in observational studies, where neither random sampling nor randomization is performed and uncontrolled sources of bias, such as confounding, selection bias and measurement error can be present (2). Through the use of informative priors (IPs) for the coefficients of interest, Bayesian framework can be a very attractive alternative (3).

In dental caries epidemiology, the dmft (DMFT) index, defined as the number of primary (perma- nent) teeth ( $t$ ) that are decayed (d), missing (m) or filled (f) because of dental caries disease, has been the most used indicator to measure the caries experiences of individuals.

During the 1970s, the prevalence of dental caries showed a substantial decrease in most industrialized countries (4-8), which stabilized from 1980 onwards (9) chiefly due to the widespread use of fluoride-containing toothpastes (10-14). As a consequence, nowadays, the $\mathrm{dmft}$ (DMFT) distribution is characterized by being very positively skewed with a high proportion of zero scores, corresponding to healthy people.

Poisson and binomial distributions are the most suitable theoretical distributions to model count data. If the data have larger variance than expected under the assumption of a Poisson/binomial distribution, an overdispersion parameter should be included to measure the variance surplus. The Poisson distribution and other Poisson-based models like the overdispersed Poisson, best known as the negative binomial (NB), the zero-inflated Poisson (ZIP) and the zero-inflated NB (ZINB), have been extensively used to model the $\mathrm{dmft}$ (DMFT) index (15-17).

Zero-inflated models can be proposed if data have excess zeros, reflecting a desirable health condition. These models constitute a mixture of a standard probability distribution for count data, representing a 'susceptible' subpopulation of children said to be at risk for a disease or condition (e.g. dental caries), and a subpopulation of 'nonsusceptible' children with only zero counts (structural zeros), who are considered to be not at risk (18).

Indeed, in proper statistical modelling of the $\mathrm{dmft}$ (DMFT) index, the bounded nature of data must be considered. In fact, the dmft (DMFT) index may range between zero and twenty (or thirtytwo), the number of teeth in the child's (adult's) mouth. In this case, the Poisson distribution is not appropriate because it is only recommended to model unbounded count data. Binomial models and other binomial-based models like overdispersed binomial, best known as beta-binomial (BB) model, the zero-inflated binomial (ZIB) and the zero-inflated $\mathrm{BB}$ (ZIBB), have to be considered instead of Poisson-like models (19-22).

Most studies on the subject follow the Frequentist estimation approach $(17,19-28)$. The Bayesian approach was proposed for Poisson-based models, like ZIP, ZINB models (29-32) and zero-inflated Poisson difference (ZIPD) models for paired count data (33), and hurdle Poisson, ZIP and zero-altered 
Poisson models for longitudinal zero-inflated count data (34).

The aim of this study was to propose Bayesian modelling of the $\mathrm{dmft}$ (DMFT) index by the application of ZIB and ZIBB models and to guide the epidemiologist in the choice of the best model depending on the presence or absence of overdispersion and excess zeros. Moreover, the effects of incorporating prior information about a model's parameter were considered.

The performance of the proposed methods was shown on the data of the Belo Horizonte Caries Prevention (BELCAP) study (16), the most frequently used for methodological purposes in dental caries research within the Frequentist statistics framework (available at http://cran.r-project. org/). Analysis of these data is not given any clinical interpretation but has only been considered as illustrative of the methodology.

\section{Methods}

Data of five variables were collected among 797 seven-year-old school children living in an urban area of Belo Horizonte (Brazil) for the evaluation of four programmes for reducing caries: oral health education, enrichment of the school diet with rice bran, rinsing with 0.2 per cent sodium fluoride solution and oral hygiene. Two additional experimental factors were considered: all the interventions together and none of the interventions. Altogether, the data set comprised a factor variable with six levels called treatment, two categorical variables indicating gender and ethnic group, the latter with 3 levels brown, white and black, and two count variables indicating the $\mathrm{dmft}$ index before and after the treatment, respectively, named begin and end. Data were recorded for the eight primary molar teeth only, so in this data set, the $\mathrm{dmft}$ index ranges between 0 and 8 . While only negative development can be expected in studies on elderly populations, in this data set, the caries state of children could improve (16). The experimental design was without replication, with every treatment randomized to only one school.

Zero-inflated binomial and zero-inflated betabinomial models were included in data analysis. Referring to the ZIB model, if $N$ is the number of teeth in the mouth, $\pi_{\mathrm{i}}$ is the probability of being structural zero and $p_{i}$ represents the probability of being caries affected at the end of the treatment for susceptible children, the probability of $\mathrm{dmft}$ counts
$\mathrm{Y}_{\mathrm{i}}, i=1,2, \ldots, n$ can be expressed as:

$$
\begin{gathered}
\operatorname{Prob}\left(\mathrm{y}_{\mathrm{i}}=0\right)=\pi_{\mathrm{i}}+\left(1-\pi_{\mathrm{i}}\right)\left(1-\pi_{\mathrm{i}}\right)^{\mathrm{N}} \\
\operatorname{Prob}\left(\mathrm{y}_{\mathrm{i}}=\mathrm{y}\right)=\left(1-\pi_{\mathrm{i}}\right)\left(\begin{array}{l}
\mathrm{N} \\
\mathrm{y}
\end{array}\right) \mathrm{p}_{\mathrm{i}}^{\mathrm{y}}\left(1-\mathrm{p}_{\mathrm{i}}\right)^{\mathrm{N}-\mathrm{y}}
\end{gathered}
$$

meaning that the overall probability of zero counts is the combined probability of zeros from two groups (nonsusceptible and susceptible children), and the probability of positive counts comes from the group of susceptible children only. The probability $\pi_{\mathrm{i}}$ and the binomial mean $p_{i}$ may depend upon covariates through appropriate link functions, which both take the form of the logit.

$$
\begin{aligned}
\operatorname{logit}\left(\pi_{i}\right)= & \alpha_{0}+\alpha_{1} \text { male }_{i}+\alpha_{2,1} \text { white }_{i}+\alpha_{2,2} \text { black }_{i} \\
& +\alpha_{3,1} \text { educ }_{i}+\alpha_{3,2} \text { all }_{i} \\
& +\alpha_{3,3} \text { enrich }_{i}+\alpha_{3,4} \text { rinse }_{i}+\alpha_{3,5} \text { hygiene }_{i} \\
\operatorname{logit}\left(p_{i}\right)= & \beta_{0}+\beta_{1} \text { male }_{i}+\beta_{2,1} \text { white }_{i}+\beta_{2,2} \text { black }_{i} \\
& +\beta_{3,1} \text { educ }_{i}+\beta_{3,2} \text { all }_{i} \\
& +\beta_{3,3} \text { enrich }_{i}+\beta_{3,4} \text { rinse }_{i}+\beta_{3,5} \text { hygiene }_{i}
\end{aligned}
$$

Through model [2], it was investigated whether there was a significant association of the $\mathrm{dmft}$ at the end of follow-up with gender, ethnicity and treatment, all considered as fixed effects defined at child level. Female, brown and none of the interventions served as reference categories to estimate the odds ratio (OR) of caries. ZIBB models could be easily expressed as well, with the necessary adaptations (19).

Results of models were described in terms of medians, 95\% CrIs and MC Error/SD of posterior distributions of ORs.

The data augmentation procedure for Bayesian estimation was applied $(30,31)$. Under a full Bayesian estimation approach, prior distributions were assumed for all parameters. The regression coefficients $\alpha$ 's and $\beta^{\prime}$ s were assumed to follow noninformative prior normal distributions with zero mean and precision $=10^{-3}$, and the overdispersion parameter was assumed to follow noninformative gamma $\left(10^{-3}, 10^{-3}\right)$. In further analysis, to incorporate prior information about $\pi_{i}$ and $\pi$, we used IPs for the intercepts $\alpha_{0}$ and $\beta_{0}$ in [2] and assigned fixed known values to the parameters of their prior distributions (called hyperparameters). As the probability of being a structural zero is always less than the probability of being caries-free $\phi(18)$, for $\alpha_{0}$ we used the normal distribution centred on mean equal to $\ln (\phi /(1-\phi))$ and truncated for values more than the mean. As the mean $\mathrm{dmft}$ in 
susceptible subpopulation is always more that the mean $\mathrm{dmft}$ in the whole population $v(18)$, for $\beta_{0}$ we used the normal distribution centred on mean equal to $\ln (v /(N-v))$ and truncated for values less than the mean. For all regression coefficients, precision was chosen equal to $10^{-1}$, and the overdispersion parameter was assumed to follow gamma $\left(10^{-1}, 10^{-1}\right)$. From oral health statistics of the Brazilian population (35), mean $\mathrm{dmft}$ for 5 -year-old children (twenty primary teeth) in 1996 was $v=3.0$ and \% of caries-free children was $\phi=0.406$ in 2003. The codes for ZIB and ZIBB models are described in the Appendix.

All analyses were performed using Winbugs 14.0 (36), a Bayesian software package which is free of charge. Sampling from posterior distribution of zero-inflated models was performed through MCMC techniques (37). The models were fitted using the Metropolis-Hastings algorithm $(38,39)$. To eliminate the influence of the starting values, an initial 10000 burn-in iterations was done. Every 10th MCMC sample thereafter was retained from the next 100000 iterations, to eliminate autocorrelation, and 10000 samples were obtained for subsequent posterior inference of the unknown parameters. Convergence of the generated samples was assessed using standard Winbugs tools (such as trace plots and autocorrelation function (ACF) plots). The Monte Carlo error (MC) by standard deviation (SD) ratio was used as quality indicator of MCMC simulations, because it measures the variability of each estimate due to the simulation. The cut-off 0.05 was considered to indicate bad performance. The deviance, defined as -2 times the log-likelihood, was used to assess goodness of fit. The lower the deviance, the better the fit.

\section{Results}

Results of the ZIB model with noninformative prior showed that, of the six interventions considered in the prevention programme, all interventions together and rinsing resulted statistically significant in predicting the probability of a child being a structural zero, with posterior ORs and 95\% CrIs, respectively, equal to 2.90 (95\% CrI: 1.45, 5.91) and 2.63 (95\% CrI: 1.39, 5.12) (data not in Table). With regard to the count part of the ZIB model (Table 1, 1st column), only education $(\mathrm{OR}=0.70,95 \%$ CrI: 0.55, 0.89), hygiene $(\mathrm{OR}=0.77$, $95 \%$ CrI: $0.60,0.97)$ and all interventions $(\mathrm{OR}=0.62$, 95\% CrI: $0.48,0.80)$ were associated with a decreased probability of a child being caries affected. The percentage of caries-free children was estimated as $28.7 \%$ (95\% CI: $25.7,31.8)$. The deviance was $\mathrm{D}_{\mathrm{ZIB}}=2198$ (95\% CI: 2123, 2290).

From the ZIBB model with noninformative prior (Table 1, 2nd column), an overdispersion parameter equal to 0.17 (95\% CI: $0.14,0.21$ ) was estimated, which seemed to provide sufficient explanation of the unobserved heterogeneity. In fact, unlike the ZIB results, none of the interventions considered in the prevention programme was associated with the probability of being a structural zero compared to the control group (data not in Table). With regard to the count part of the $\mathrm{ZIBB}$, education $(\mathrm{OR}=0.76$, 95\% CrI: 0.59, 0.99), rinsing (OR=0.61, 95\% CrI: $0.47,0.80)$, hygiene $(\mathrm{OR}=0.65,95 \%$ CrI: $0.49,0.86)$ and all interventions (OR $=0.46,95 \%$ CrI: $0.35,0.62)$ protected children from being caries affected. Being male increased the probability of being caries diseased ( $\mathrm{OR}=1.19,95 \% \mathrm{CrI}: 1.01,1.42)$. The percentage of caries-free children was estimated as $27.6 \%$ (95\% CI: 24.8, 30.5). The deviance was $\mathrm{D}_{\mathrm{ZIBB}}=2152$ (95\% CI: 2070, 2237). After incorporating IPs (Table 1, 3rd column), ZIB model estimates were not influenced, while ZIBB models reduced deviance $\left(D_{\text {ZIBB_IP }}=2136\right.$ (95\% CrI: 2048, 2225)) and showed the association with all interventions and rinsing only. All models agreed in estimating the mean dmft as 1.84 (95\% CrI: 1.73, 1.96).

\section{Discussion}

The long debate in literature on caries data analysis dates back to the pivotal study of Böhning et al. (16), who first proposed the use of the ZIP model to take account of excess zeros. In the wake of this study, ZIB and ZIBB models were proposed (19, 22) to take into account the bounded nature of the $\mathrm{dmft}$ index, and the use of covariates in the count part of zero-inflated models was advocated. In the present study, we developed ZIB and ZIBB models within a Bayesian framework and we also modelled prior information about parameters.

In case of zero-inflated modelling, the advantages of the Bayesian compared to the classical approach can be highlighted. In classical methods such as maximum likelihood (ML), parameter estimates are found through numerical optimization, which can be computationally intensive in the presence of many unknown parameter values. Alternatively, Bayesian parameter estimates are found by drawing realizations from the posterior 
Table 1. ZIB and ZIBB models for BELCAP study: medians, 95\% credibility intervals and MC error/SD of posterior distributions of odds ratios of being caries affected in susceptible children

\begin{tabular}{|c|c|c|c|c|c|c|}
\hline & \multicolumn{2}{|c|}{$\begin{array}{l}\mathrm{ZIB}^{\mathrm{b}} \text { with noninformative } \\
\text { prior }\end{array}$} & \multicolumn{2}{|c|}{$\begin{array}{l}\mathrm{ZIBB}^{\mathrm{b}} \text { with noninformative } \\
\text { prior }\end{array}$} & \multicolumn{2}{|c|}{$\begin{array}{l}\mathrm{ZIBB}^{\mathrm{b}} \text { with informative } \\
\text { prior }\end{array}$} \\
\hline & ORs $95 \%$ CrI & MC Error/SD & OR 95\% CrI & MC Error/SD & OR 95\% CrI & MC Error/SD \\
\hline Intercept & $0.49(0.40,0.60)$ & 0.041 & $0.37(0.29,0.46)$ & 0.045 & $0.38(0.29,0.50)$ & 0.041 \\
\hline \multicolumn{7}{|l|}{ Intervention $^{\mathrm{a}}$} \\
\hline $\begin{array}{l}\text { Education } \\
\text { versus none }\end{array}$ & $0.70(0.55,0.89)$ & 0.030 & $0.76(0.59,0.99)$ & 0.033 & $0.75(0.56,1.00)$ & 0.035 \\
\hline $\begin{array}{l}\text { Enrichment } \\
\text { versus none }\end{array}$ & $0.92(0.73,1.13)$ & 0.030 & $0.88(0.68,1.15)$ & 0.034 & $0.89(0.66,1.22)$ & 0.037 \\
\hline $\begin{array}{l}\text { Rinsing } \\
\text { versus none }\end{array}$ & $0.82(0.66,1.03)$ & 0.031 & $0.61(0.47,0.80)$ & 0.034 & $0.65(0.47,0.96)$ & 0.043 \\
\hline $\begin{array}{l}\text { Hygiene } \\
\text { versus none }\end{array}$ & $0.77(0.60,0.97)$ & 0.028 & $0.65(0.49,0.86)$ & 0.032 & $0.65(0.48,0.91)$ & 0.036 \\
\hline $\begin{array}{l}\text { All versus } \\
\text { none }\end{array}$ & $0.62(0.48,0.80)$ & 0.027 & $0.46(0.35,0.62)$ & 0.033 & $0.48(0.34,0.70)$ & 0.039 \\
\hline \multicolumn{7}{|l|}{ Gender $^{\mathrm{a}}$} \\
\hline $\begin{array}{l}\text { Male versus } \\
\text { female }\end{array}$ & $1.12(0.97,1.29)$ & 0.027 & $1.19(1.01,1.42)$ & 0.030 & $1.18(0.98,1.42)$ & 0.033 \\
\hline \multicolumn{7}{|l|}{ Ethnicity $^{\mathrm{a}}$} \\
\hline $\begin{array}{l}\text { White versus } \\
\text { brown }\end{array}$ & $0.13(0.96,1.31)$ & 0.028 & $1.13(0.94,1.36)$ & 0.032 & $1.14(0.881 .44)$ & 0.042 \\
\hline $\begin{array}{l}\text { Black versus } \\
\text { brown }\end{array}$ & $0.92(0.73,1.16)$ & 0.021 & $0.82(0.62,1.08)$ & 0.028 & $0.84(0.61,1.19)$ & 0.037 \\
\hline Overdispersion & & & $0.17(0.14,0.21)$ & 0.018 & $0.16(0.12,0.20)$ & 0.035 \\
\hline Deviance & $2198(2123,2290)$ & 0.016 & $2152(2070,2237)$ & 0.013 & $2136(2048,2225)$ & 0.019 \\
\hline $\begin{array}{l}\text { Estimated } \\
\text { mean } \mathrm{dmft}\end{array}$ & $1.85(1.75,1.96)$ & 0.011 & $1.84(1.73,1.96)$ & 0.018 & $1.84(1.73,1.96)$ & 0.011 \\
\hline $\begin{array}{l}\text { Estimated } \% \\
\text { of zeros }\end{array}$ & $28.7(25.7,31.8)$ & 0.009 & $27.6(24.8,30.5)$ & 0.018 & $27.8(25.0,30.8)$ & 0.015 \\
\hline
\end{tabular}

a Reference categories: 'none' for intervention, 'female' for gender, 'brown' for ethnicity.

${ }^{\mathrm{b}}$ All covariates were included to model zero inflation.

Abbreviations: ZIB, zero-inflated binomial; ZIBB, zero-inflated beta-binomial.

distribution. Within large data set, the two methods are shown to be equivalent through the Bernstein-von Mises theorem (40). This property allows Bayesian methods to be used in place of classical methods, under certain conditions, which are asymptotically similar and may have significant computational advantages.

Furthermore, the advantages of Bayesian analysis include user-friendly interpretation of estimated parameters, practical estimation of any function of parameters or predictive values, reduced small-sample bias compared to ML procedures $(30,41)$ and incorporation of prior information, if not for all the coefficients, at least for the confounding ones (1). The greatest flexibility of Winbugs software in adapting the model assumptions compared to other statistical packages (42), in addition to its availability and popularity, is that it can balance the lower computational speed caused by MCMC sampling.

To show the potential of Bayesian analysis in statistical modelling of the $\mathrm{dmft}$ index, we used the BELCAP data set, which is easily accessible but affected by a weak study design, with only one treatment arm randomized to one school.

The practice for Bayesian data analysis starts with considering noninformative priors as 'reference models' to be used as a standard of comparison or starting point in place of the proper, informative prior distributions that would be appropriate for a full Bayesian analysis $(43,44)$. After the model has been fitted with noninformative prior, one should look at the posterior distribution and see whether it makes sense. If the posterior distribution does not make sense, this implies that additional prior knowledge is available that has not been included in the model and that contradicts the assumptions of the prior distribution that has been used. It is then appropriate to go back and alter the prior distribution to be more consistent with this external knowledge (45).

In the first phase of our study, we used noninformative diffuse priors to extend the range of possible values for each parameter. The drawback of this choice could be the increase in the width of CrIs and the consequent loss in precision of estimated 
parameters. Our estimates had about the same accuracy and precision as likelihood-based estimates (19). With large sample size, Bayesian estimates are much more driven by the observed data and less by the priors and they correlate very closely with ML estimates, as they have the same asymptotic normal distribution.

On the base of the mean dmft or the percentage of caries-free children, our models were shown to be very similar each other. The deviance criterion indicated the best-fit model as the ZIBB, estimating higher variability than that one predicted by the ZIB model. Through Bayesian analysis, it was possible to model overdispersion easily, using a noninformative gamma distribution in the MCMC algorithm.

In the second phase of our study, we used IPs for the intercepts of inflation and count components of the models. From the point of view of the epidemiological study, this means giving prior information about both the odds of being a structural zero dmft and the odds of being caries affected in the subpopulation of susceptible children, when all covariates are null. After incorporating IPs, ZIBB model estimates were affected in such a way that education and gender were no longer associated with the probability of being caries affected. The introduction of prior information can have significant effects on the parameters of the model through hyperparameters, which can control the amount of noise in a regression model, the degree to which various input variables are relevant, and the magnitudes of different additive components of a model (46). Hence, prior information should be adequately supported by literature and should receive the consensus of the relevant scientific community.

The overdispersion parameter can expound why IPs affected only ZIBB models, while ZIB estimates remained unchanged. In fact, the overdispersion parameter explains a significant proportion of the total variability of the response that the ZIB model is not able to explain. When adding further information through IPs, some variables become redundant in the ZIBB but not in the ZIB model.

One limitation of our study was associated with the choice of the goodness-of-fit statistics. In fact, the deviance information criteria (DIC) obtained by incorporating into the deviance a penalization factor for the number of estimated parameters (47) may not be a viable option when posterior distributions are extremely skewed or bimodal (36), as it is in case of zero-inflated mod- els. Moreover, the default DIC option in Winbugs was not available for the models presented in this article. In any case, with a large sample size, the penalization factor is negligible with regard to the deviance. There are a variety of DIC alternatives, but many of these are more computationally challenging than the default provided by Winbugs (34). While its original formulation is appropriate in most generalized linear modelling problems, it fails in other contexts (36).

Bayesian estimation could be suggested as an alternative to Frequentist methods in the modelling of the $\mathrm{dmft}$ index. In Bayesian analysis, the probability of being a structural zero and the probability of being caries affected related to covariates could be investigated by checking whether the hypothesized value lies in the central region or in the tails of the posterior distribution. In this case, the corresponding CrIs could give the epidemiologist an idea about whether his/her assumption could be plausible or not. Moreover, Bayesian models could give the opportunity to include his/her own personal beliefs about the phenomenon under investigation.

\section{References}

1. Dunson DB. Commentary: practical advantages of Bayesian Analysis of epidemiologic data. Am J Epidemiol 2001;153(12):1222-6.

2. Greenland S. Bayesian perspectives for epidemiological research: I Foundations and basic methods. Int J Epidemiol 2006;35:765-75.

3. Greenland S. Multiple-bias modeling for analysis of observational data (with discussion). J R Stat Soc 2005;168:267-308.

4. Frencken JE, Kalsbeek H, Verrips GH. Has the decline in dental caries been halted? Changes in caries prevalence amongst 6- and 12-year-old children in Friesland, 1973-1988. Int Dent J 1990;40:225-30.

5. Truin GJ, König KG, De Vries HCB, Mulder J, Plasschaert AJM. Trends in caries prevalence in 5-, 7- and 11-year-old schoolchildren in The Hague between 1969 and 1989. Caries Res 1991;25:462-7.

6. Nugent ZJ, Pitts NB. Patterns of change and results overview 1985/6-1995/6 from the British Association for the Study of Community Dentistry (BASCD) coordinated National Health Service surveys of caries prevalence. Community Dent Health 1997;14:30-54.

7. Downer MC. The changing pattern of dental disease over 50 years. Br Dent J 1998;185:36-41.

8. Truin GJ, König KG, Bronkhorst EM, Frankenmolen F, Mulder J, van't Hof MA. Time trends in caries experience of 6- and 12-year-old children of different socioeconomic status in The Hague. Caries Res 1998;32:1-4.

9. Truin GJ, König KG, Kalsbeek H. Trends in dental caries in the Netherlands. Adv Dent Res 1993;7:15-8. 
10. Pizzo G, Piscopo MR, Pizzo I, Giuliana G. Community water fluoridation and caries prevention: a critical review. Clin Oral Invest 2007;11:189-93.

11. Bratthall D. Estimation of global DMFT for 12 yearolds in 2004. Int Dent J 2005;55:370-2.

12. Solinas G, Campus G, Maida C, Sotgiu G, Cagetti $M G$, Lesaffre E et al. What statistical method should be used to evaluate risk factors associated with dmfs index? Evidence from the National Pathfinder Survey of 4-years-old Italian children. Community Dent Oral Epidemiol 2009;37:539-46.

13. Petersen PE, Christensen LB. Dental health status and development trends among children and adolescents in Greenland. Int J Circumpolar Health 2006;65:35-44.

14. Pitts NB, Boyles J, Nugent ZJ, Thomas N, Pine CM. The dental caries experience of 11-year-old children in Great Britain: surveys coordinated by the British Association for the Study of Community Dentistry in 2004/2005. Community Dent Oral Epidemiol. 2006;23:44-57.

15. Lewsey J, Thomson W. The utility of the zeroinflated Poisson and zero-inflated negative binomial models: a case study of cross-sectional and longitudinal DMF data examining the effect of socio-economic status. Community Dent Oral Epidemiol 2004;32:18389.

16. Böhning D, Dietz E, Schlattmann P, Mendoca L, Kirchner U. The zero-inflated Poisson model and the decayed, missing and filled teeth index in dental epidemiology. J R Stat Soc Ser A Stat. 1999;162(2):195209.

17. Ridout M, Demetrio CGB, Hinde J. Models for count data with many zeros. Presented at the XIXth International Biometric Conference, Cape Town, South Africa, December 1998; (http://www.kent.ac.uk/ smsas/personal/msr/webfiles/zip/ibc_fin.pdf).

18. Preisser JS, Stamm JW, Long DL, Kinkade ME. Review and recommendations for zero-inflated count regression modeling of dental caries indices in epidemiological studies. Caries Res 2012;46(4):41323.

19. Gilthorpe MS, Frydenberg M, Cheng Y, Baelum V. Modelling count data with excessive zeros: the need for class prediction in zero-inflated models and the issue of data generation in choosing between zeroinflated and generic mixture models for dental caries data. Stat Med 2009;28:3539-53.

20. Hall DB. Zero-inflated Poisson and binomial regression with random effects: a case study. Biometrics. 2000;56(4):1030-9.

21. Vieira AMC, Hinde JP, Demetrio CGB. Zero-inflated proportion data models applied to a biological control assay. J Appl Stat 2000;27(3):373-89.

22. Skrondal A, Rabe-Hesketh S. Generalized Latent Variable Modeling: Multilevel, Longitudinal and Structural Equation Models. Boca Raton, FL: Chapman \& Hall/CRC; 2004.

23. Yau K, Lee A. Zero-inflated Poisson regression with random effects to evaluate an occupational injury prevention programme. Stat Med 2001;20:2907-20.

24. Hur K, Hedeker D, Henderson WG, Khuri S, Daley J. Modeling clustered count data with excess zeros in health care outcomes research. Health Serv Outcomes Res Methodol 2002;1(1):5-20.
25. Lee AH, Wang K, Scott JA, Yau KKW, McLachlan GJ. Multi-level zero-inflated poisson regression modelling of correlated count data with excess zeros. Stat Methods Med Res 2006;15(1):47-61.

26. Moghimbeigi A, Eshraghian MR, Mohammad K, McArdle B. Multilevel zero-inflated negative binomial regression modeling for overdispersed count data with extra zeros. J Appl Stat 2008;35(10):1193202.

27. Burnside G, Pine CM, Williamson PR. The application of multilevel modelling to dental caries data. Stat Med 2007;26:4139-49.

28. Molenberghs G, Verbeke G, Demetrio CGB, Vieira AMC. A family of generalized linear models for repeated measures with normal and conjugate random effects. Statist Sci 2010;25(3):325-47.

29. Mwalili SM, Lesaffre E, Declerck D. The zero-inflated negative binomial regression model with correction for misclassification: an example in caries research. Stat Methods Med Res 2008;17(2):123-39.

30. Ghosh SK, Mukhopadhyay P, Lu JC. Bayesian analysis of zero inflated regression models. Calcutta, India: Invited talk delivered at Indian Statistical Institute, Applied Statistics Unit; 1999.

31. Ghosh SK, Mukhopadhyay P, Lu JC. Bayesian analysis of zero-inflated regression models. J Stat Plan Inference 2006;136:1360-75.

32. Bhattacharya A, Clarke BS, Datta GS. A Bayesian test for excess zeros in a zero-inflated power series distribution. Inst Math Stat Collect 2008;1:89-104.

33. Karlis D, Ntzoufras I. Bayesian analysis of the differences of count data. Stat Med 2006;25:1885-905.

34. Neelon BH, O'Malley AJ, Normand SLT. A Bayesian model for repeated measures zero-inflated count data with application to outpatient psychiatric service use. Stat Model 2010;10(4):421-39.

35. WHO Oral Health Country/Area Profile Programme (CAPP). (http://www.mah.se/CAPP/Country-OralHealth-Profiles/AMRO/Brazil/Oral-Diseases/DentalCaries/)

36. Spiegelhalter DJ, Thomas A, Best NG, Lunn D eds. WinBUGS Version 1.4 Users Manual. Cambridge, UK: MRC Biostatistics Unit, Publisher; 2003. (http:/ / www.mrc-bsu.cam.ac.uk/bugs/).

37. Gilks W, Richardson S, Spiegelhalter D. Markov Chain Monte Carlo in Practice. Interdisciplinary Statistics. Suffolk, UK: Chapman \& Hall; 1996.

38. Tanner M. Tools for statistical inference. New York, USA: Springer; 1993.

39. Gelman A, Carlin JB, Stern HS, Rubin DB. Bayesian data analysis. London, UK: Chapman \& Hall/CRC; 1995.

40. Train KE. Discrete Choice Methods with Simulation. Cambridge, UK: Cambridge University Press; 2003.

41. Ntzoufras I. Bayesian Modeling Using Winbugs. Hoboken, New Jersey, USA: John Wiley \& Sons, Inc; 2009.

42. Li B, Lingsma HF, Steyerberg EW, Lesaffre E. Logistic random effects regression models: a comparison of statistical packages for binary and ordinal outcomes. BMC Med Res Methodol 2011;11:77.

43. Bernardo JM. Reference posterior distributions for Bayesian inference (with discussion). J R Stat Soc Ser B 1979;41:113-47. 


\section{Matranga et al.}

44. Kass RE, Wasserman L. The selection of prior distributions by formal rules. J Am Stat Assoc 1996;91:1343-70.

45. Gelman A, Hill J. Data analysis using Regression and Multilevel/Hierarchical Models. Cambridge, NY: Cambridge University Press Publisher; 2007

46. Neal RM. Monte Carlo Implementation of Gaussian Process Models for Bayesian Regression and Classifi- cation. Technical Report 9702. Toronto, Canada: Department of Statistics, University of Toronto; 1997 (http:/ /arxiv.org/pdf/physics/9701026.pdf).

47. Spiegelhalter DJ, Best NG, Carlin BP, Van Der Linde A. Bayesian measures of model complexity and fit. J R Stat Soc Ser B 2002;64:583-639.

\section{Appendix}

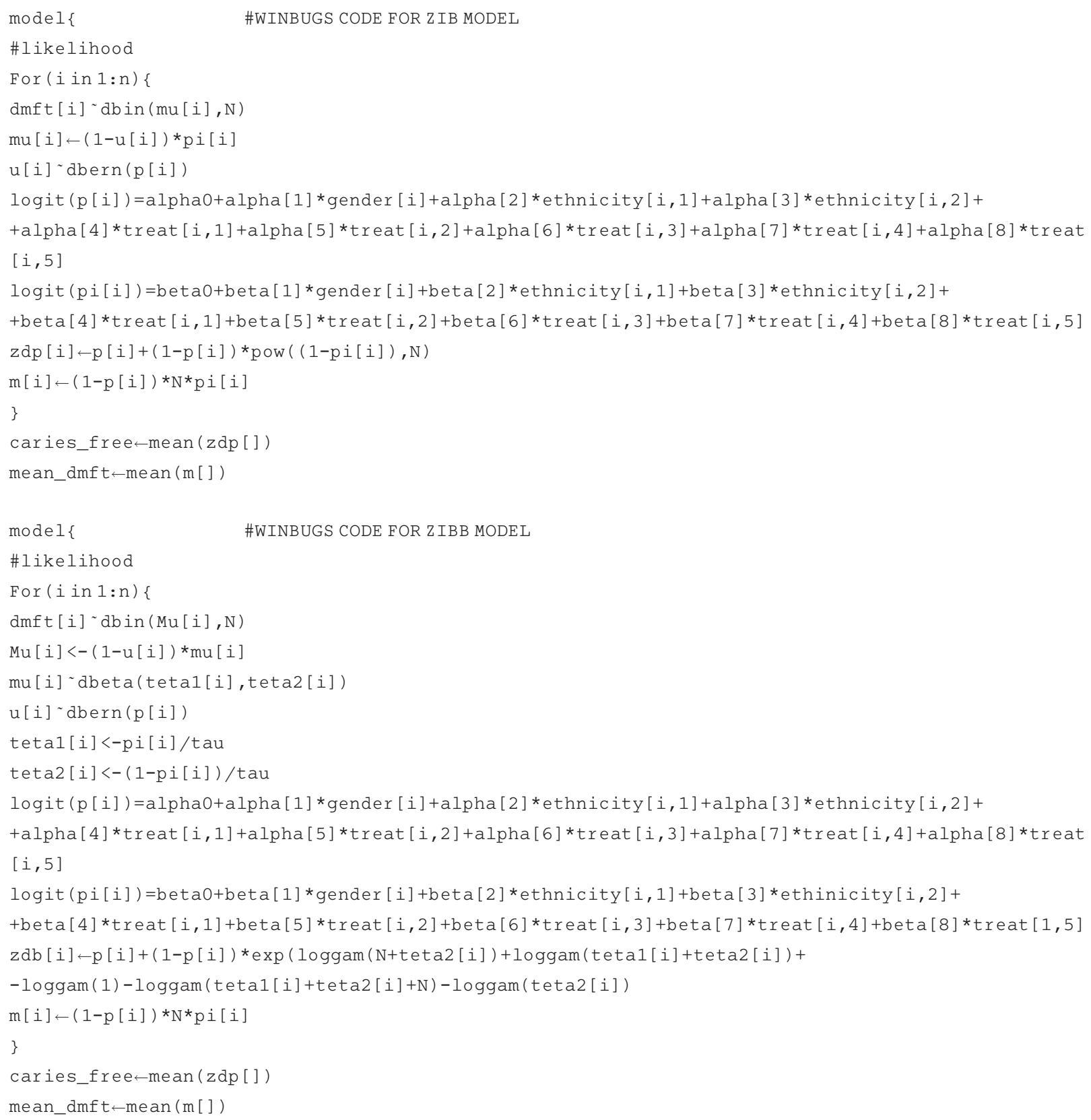

